\title{
Simple Structural Econometrics of Price Elasticity
}

\author{
Catherine Cazals \\ University of Toulouse \\ (GREMAQ and IDEI) \\ Patrick Fève* \\ University of Toulouse \\ (CNRS-GREMAQ and IDEI)
}

Frédérique Fève

University of Toulouse

(GREMAQ and IDEI)

Jean-Pierre Florens

University of Toulouse

(IUF, GREMAQ and IDEI)

\begin{abstract}
Abstact
The identification of demand parameters from individual data may be very difficult due to the lack of price variation. The aim of this paper is to deliver a simple methodology for the
\end{abstract}


a structural model of demand. Using the restrictions created by this structural model, we consider the identification of the structural parameters in various cases. We first show that the structural parameters are not identified in the one period case. Nevertheless, the identification can be obtained using different time periods. The motivation of such an approach is that, if preferences and the distribution of tastes remain constant across time, exogenous prices changes allows to identify the structural parameters. We exploit this idea and we show in a series of examples that the parameters that summarize preferences can be easily identified.

The paper is organized as follows. In a first section, we present a simple structural model of demand and we discuss some modeling issues. In a second section, we show that the structural parameters are not identified when data concern a single period, apart from particular restrictions on the distribution of the heterogeneity factor. In a third section, we present - as a benchmark- the structural model in the two period case and we discuss a series of examples wherein the structural parameters and thus the price elasticity of demand are identified. A last section offers some concluding remarks.

\section{A simple structural model}

The structural model is devoted to the identification of structural parameters that summarize consumers'preferences, considering the supply side as given. The quantity of a homogeneous $^{1}$ good used by a consumer is denoted $q$ and the associated price is $p$. The preferences are described by the following linear-quadratic utility function

$$
U(q)=\frac{a}{2} q^{2}+q \theta+X_{o}
$$

where $a<0$ is the same for all consumers. $\theta$ is assumed to be specific to each consumer and randomly generated. Consumers thus differ in their preference parameter $\theta$. In what follows, we consider that $\theta$ is observed only by the consumer. For the simplicity of the presentation, we omit for the moment individual $i$ and time $t$ indexes. $X_{o}$ represents all the other goods and enters linearly into the utility function. The budget constraint is given by $p q+X_{o}=R$, where $R$ is the disposable income of consumers and the price of $X_{o}$ is normalized to one. After substitution for $X_{o}$, the utility function rewrites - up to a constant term -

$$
U(q)=\frac{a}{2} q^{2}+q \theta-p q
$$

The first order condition of the maximization of utility yields the demand equation

$$
q=\beta(p-\theta)
$$

where $\beta=1 / a<0$. The demand is a linear function of the difference between the price $p$ and the random variable $\theta$ that represents shifts in preferences. Note that the demand function introduces a non-additive error term $\theta$.

Let us consider now the more general case of many consumers $i=1, \ldots, n$ for different time periods $t=1, \ldots, T$. It is worth noting that the price $p$ is common to all consumers. In order to determine total demand and expenditure, we have now to specify the random

\footnotetext{
${ }^{1}$ Although excessively simplified, this structural model delivers the basis of our identification principle. See Fève, Fève and Florens (2003) for several extensions of this simple model.
} 
variable - or heterogeneity factor $-\theta(i, t)$ for each consumer $i$ at any period $t$. This random variable can be specified as follows

$$
\theta_{i, t}=\theta_{i}+\varepsilon_{t}
$$

where $\theta_{i}$ is a random variable specific to each consumer and $\varepsilon_{t}$ is an independent and serially uncorrelated aggregate random variable. We can thus extend the specification of $\theta_{i, t}$ to the case of an exogenous trend. In such a case, the exogenous trend can account for growth in demand independently from price variations: ${ }^{2}$

$$
\theta_{i, t}=\mu^{t-1}\left(\theta_{i}+\varepsilon_{t}\right)
$$

For $\mu>1$, the mean and the variance of $\theta_{i, t}$ will increase over time.

Given these specifications of the heterogeneity factor, demand and expenditure can be written in the following more compact form

$$
\begin{aligned}
x_{i, t} & =\alpha_{t}-\beta \theta_{i, t} \\
y_{i, t} & =\gamma_{t}-\alpha_{t} \theta_{i, t}
\end{aligned}
$$

The parameter $\alpha_{t}=\beta p_{t}$ is negative and varies over periods following a change in price. As previously mentioned, the parameter $\beta=1 / a$ is negative and time invariant. Finally, the parameter $\gamma_{t}=\beta p_{t}^{2}$ is negative and changes with the square of the price.

Equations (1)-(2) can thus be used in order to identify and estimate the structural parameters that summarize preferences. The structural model imposes cross-equations restrictions as the parameter $\alpha_{t}$ enter both in (1) and (2). The idea is now to take advantage from these restrictions in order to recover the structural parameters. In what follows, we are interested in the identification of the price elasticity of demand from the structural parameters. The prices elasticities at period $t$ of a representative consumer is given by:

$$
\mathcal{E}_{t}=\frac{\partial E\left(x_{i, t}\right)}{\partial p_{t}} \frac{p_{t}}{E\left(x_{i, t}\right)} \equiv \frac{\alpha_{t}}{E\left(x_{i, t}\right)}
$$

\section{Identification in the one period case}

In order to illustrate the difficulties about the identification of structural parameters, we first introduce the simple case where data are only available for a single period. So, we discard from (1) and (2) the time index. The demand and expenditure are thus defined by $x_{i}=\alpha-\beta \theta_{i}$ and $y_{i}=\gamma-\alpha \theta_{i}$. We denote $E\left(\theta_{i}\right)=\theta_{o}$ and $V\left(\theta_{i}\right)=V_{o}$. In this simple static case, we do not impose any additional restrictions either on the demand and expenditure functions or on the heterogeneity factor $\theta_{i}$. Moments conditions on demand and expenditure are the $E\left(x_{i}\right)=\alpha-\beta \theta_{0}, E\left(y_{i}\right)=\gamma-\alpha \theta_{0}, V\left(x_{i}\right)=\beta^{2} V_{o}, V\left(y_{i}\right)=$ $\alpha^{2} V_{o}$ and $\operatorname{Cov}\left(x_{i}, y_{i}\right)=\beta \alpha V_{o}$. The set of structural parameters to be estimated is $\varphi=$ $\left\{\alpha, \gamma, \beta, \theta_{0}, V_{o}\right\}$. The number of structural parameters is equal to the number of moments, i.e. the number of identifying conditions. So, necessary conditions for identification appears at a first glance verified. The following proposition states the identification of the structural parameters cannot be obtained using these moments condition in the static case.

\footnotetext{
${ }^{2}$ We present the case of an exponential growth. In the case of a linear trend, we have $\theta_{i, t}=\mu(t-$ 1) $\left(\theta_{i}+\varepsilon_{t}\right)$. Note that in a two period case, these two specifications provide the same results.
} 
Proposition 1 The five structural parameters $\varphi=\left\{\alpha, \gamma, \beta, \theta_{0}, V_{o}\right\}$ cannot be identified from the five moments conditions.

The identification problem comes from the covariance between demand and expenditure that does not provide any useful information for identification. In the single period case, Proposition 1 shows that we cannot take advantage from the cross-equations restriction implied by the structural model.

The identification can be obtained when particular assumptions about the distribution of the heterogeneity factor $\theta_{i}$ are formulated. The basic idea is to introduce an additional restriction on the moments using the distribution of $\theta_{i}$. Let us consider two examples where the first two moments of $\theta_{i}$ depend on a single parameter. As we are primary interested in the identification of price elasticities, we only focus on the parameter $\alpha$.

Example 2.1 Let $\theta_{i} \sim U[0, b]$ with $b>0$. The mean and the variance of $\theta_{i}$ are $E\left(\theta_{i}\right)=b / 2$ and $V\left(\theta_{i}\right)=b^{2} / 12 \equiv E\left(\theta_{i}\right)^{2} / 3$. It follows that $E\left(x_{i}\right)=\alpha-\beta b / 2$ and $V\left(x_{i}\right)=\beta^{2} b^{2} / 12$. We deduce the standard error of demand $\sigma_{x_{i}}=-\beta b /(2 \sqrt{3})$ and after substitution into the mean of demand, we obtain $\alpha=E\left(x_{i}\right)-\sqrt{3} \sigma_{x_{i}}$. We thus deduce the price elasticity $\mathcal{E}=1-\sqrt{3} \sigma_{x_{i}} / E\left(x_{i}\right)$.

Example 2.2 Let $\theta_{i} \sim f_{\theta_{o}}$ where $f$ is an exponential distribution of parameter $\theta_{o}>0$. The moments are $E\left(\theta_{i}\right)=\theta_{o}$ and $V\left(\theta_{i}\right)=\theta_{o}^{2}$. It follows that $E\left(x_{i}\right)=\alpha-\beta \theta_{o}$ and $V\left(x_{i}\right)=\beta^{2} \theta_{o}^{2}$. We obtain $\sigma_{x_{i}}=-\beta \theta_{o}$ and after substitution into the mean of demand, we obtain $\alpha=E\left(x_{i}\right)-\sigma_{x_{i}}$. The price elasticity is thus given by $\mathcal{E}=1-\sigma_{x_{i}} / E\left(x_{i}\right)$.

It is worth noting that this approach relies on an arbitrary choice about the distribution of the heterogeneity factor. Moreover, such an approach is not robust. Examples 1 and 2 can be used in order to illustrate this lack of robustness. Suppose that the heterogeneity factor is exponentially distributed, but the econometrician wrongly assumes that $\theta_{i}$ is uniformly distributed. It follows immediately that the estimate of $\alpha$ is biased. The bias is equal to $(1-\sqrt{3}) \sigma_{\theta}$ and it increases with the variance of $\theta_{i}$.

\section{Identification in the two period case}

Another - less arbitrary and more robust - way to obtain identification is to take into account for different time periods. For example, Brown and Rosen (1982) and Kahn and Lang (1988) consider the hedonic price model either with multimarket data with a single period data or single market with multiperiod data. The motivation of such an approach is that if preferences and the distribution of tastes remains stable, exogenous prices changes over periods allow to identify the structural parameters of demand. Although unexplained (see Ekeland, Heckman and Nesheim (2002) and (2003) for a critical survey), shifts over time in technology and thus in price offer an opportunity to identify the structural parameters that summarize preferences. We exploit this idea for various representations of the heterogeneity factor $\theta_{i, t}$ and we show in a series of examples that the identification of the structural parameters can be easily obtained from cross-equations 
restrictions on demand and expenditure over time. We consider the structural model in a two period case $t=1,2$. As we are interested in the identification of price elasticities, we only study the identification of the parameters $\alpha_{1}$ and $\alpha_{2}{ }^{3}$

\section{Example 3.1 Heterogeneity factor only}

The heterogeneity factor only depends on an individual effect $\theta_{i}$. Demand and expenditure are given by $x_{i, t}=\alpha_{t}-\beta \theta_{i}$ and $y_{i, t}=\gamma_{t}-\alpha_{t} \theta_{i}$, where $t=1,2$. The moments conditions are $E\left(x_{i, 1}\right)=\alpha_{1}-\beta \theta_{0}, E\left(x_{i, 2}\right)=\alpha_{2}-\beta \theta_{0}, \operatorname{Cov}\left(x_{i, 1}, y_{i, 1}\right)=\beta \alpha_{1} V_{o}, \operatorname{Cov}\left(x_{i, 2}, y_{i, 2}\right)=\beta \alpha_{2} V_{o}$. First subtract the average demand over the two periods,

$$
E\left(x_{i, 2}\right)-E\left(x_{i, 1}\right) \equiv E\left(x_{i, 2}-x_{i, 1}\right)=\alpha_{2}-\alpha_{1}
$$

in order to eliminate the individual effects and divide the covariance of demand and expenditure over the two periods

$$
\frac{\operatorname{Cov}\left(x_{i, 2}, y_{i, 2}\right)}{\operatorname{Cov}\left(x_{i, 1}, y_{i, 1}\right)}=\frac{\alpha_{2}}{\alpha_{1}}
$$

Equations (4) and (5) allow to identify the two parameters $\alpha_{1}$ and $\alpha_{2}$ :

$$
\begin{aligned}
\alpha_{1} & =\frac{\operatorname{Cov}\left(x_{i, 1}, y_{i, 1}\right)}{\operatorname{Cov}\left(x_{i, 2}, y_{i, 2}\right)-\operatorname{Cov}\left(x_{i, 1}, y_{i, 1}\right)} E\left(x_{i, 2}-x_{i, 1}\right) \\
\alpha_{2} & =\frac{\operatorname{Cov}\left(x_{i, 2}, y_{i, 2}\right)}{\operatorname{Cov}\left(x_{i, 2}, y_{i, 2}\right)-\operatorname{Cov}\left(x_{i, 1}, y_{i, 1}\right)} E\left(x_{i, 2}-x_{i, 1}\right)
\end{aligned}
$$

Using (3), we deduce the price elasticity for each period

$$
\begin{aligned}
\mathcal{E}_{1} & =\frac{\operatorname{Cov}\left(x_{i, 1}, y_{i, 1}\right)}{\operatorname{Cov}\left(x_{i, 2}, y_{i, 2}\right)-\operatorname{Cov}\left(x_{i, 1}, y_{i, 1}\right)} \frac{E\left(x_{i, 2}-x_{i, 1}\right)}{E\left(x_{i, 1}\right)} \\
\mathcal{E}_{2} & =\frac{\operatorname{Cov}\left(x_{i, 2}, y_{i, 2}\right)}{\operatorname{Cov}\left(x_{i, 2}, y_{i, 2}\right)-\operatorname{Cov}\left(x_{i, 1}, y_{i, 1}\right)} \frac{E\left(x_{i, 2}-x_{i, 1}\right)}{E\left(x_{i, 2}\right)}
\end{aligned}
$$

In this simple example, the price elasticity reduces to

$$
\begin{aligned}
\mathcal{E}_{1} & =\frac{E\left(x_{i, 2}-x_{i, 1}\right) / E\left(x_{i, 1}\right)}{\left(p_{2}-p_{1}\right) / p_{1}} \\
\mathcal{E}_{2} & =\frac{E\left(x_{i, 2}-x_{i, 1}\right) / E\left(x_{i, 2}\right)}{\left(p_{2}-p_{1}\right) / p_{2}}
\end{aligned}
$$

The structural model provides here a simple formula for the price elasticity, i.e. the relative change in average demand divided by the relative change in price.

\footnotetext{
${ }^{3}$ The remaining parameters are easily identified from the other moment conditions.
} 
Example 3.2 Heterogeneity factor with aggregate shocks

In this second example, we add an unobservable random aggregate variable in the heterogeneity factor. The demand and expenditure rewrite $x_{i, t}=\alpha_{t}-\beta \theta_{i}-\beta \varepsilon_{t}$ and $y_{i, t}=\gamma_{t}-\alpha_{t} \theta_{i}-\alpha_{t} \varepsilon_{t}$, where $E\left(\varepsilon_{t}\right)=0$ and $V\left(\varepsilon_{t}\right)=\sigma^{2}$. The moments conditions are $E\left(x_{i, 1}\right)=\alpha_{1}-\beta \theta_{0}, E\left(x_{i, 2}\right)=\alpha_{2}-\beta \theta_{0}, \operatorname{Cov}\left(x_{i, 1}, y_{i, 1}\right)=\beta \alpha_{1}\left(V_{o}+\sigma^{2}\right)$ and $\operatorname{Cov}\left(x_{i, 2}, y_{i, 2}\right)=$ $\beta \alpha_{2}\left(V_{o}+\sigma^{2}\right)$. Accounting for the aggregate shock does not modify the identification of the parameters $\alpha_{1}$ and $\alpha_{2}$ and the formula are the same than in example 3.1.

Example 3.3 Heterogeneity factor with trend.

We consider now that the heterogeneity factor evolves exogenously over periods. This model allows to reproduce situations where both the mean and the variance of demand increase - or decrease - over time. The heterogeneity factor evolves as follows

$$
\theta_{i, t}=\mu^{t-1} \theta_{i}
$$

The parameter $\mu$ represents the exogenous growth factor in preferences. The total demand rewrites $x_{i, t}=\alpha_{t}-\beta \mu^{t-1} \theta_{i}$. We can thus decompose the change in demand in two components:

$$
\underbrace{\left(x_{i, t+1}-x_{i, t}\right)}_{\text {change in demand }}=\underbrace{\left(\alpha_{t+1}-\alpha_{t}\right)}_{\text {change in price }}-\underbrace{\beta \theta_{i} \mu^{t-1}(\mu-1)}_{\text {change in preferences }}
$$

When $\mu=1$, the change in demand only results from the change in price. When $\mu \neq 1$, the change in preferences amounts to the change in demand when the price remains constant over time $\left(\alpha_{t+1}=\alpha_{t}\right)$. This growth factor is normalized to one in the first period. The moments are $E\left(x_{i, 1}\right)=\alpha_{1}-\beta \theta_{0}, E\left(x_{i, 2}\right)=\alpha_{2}-\beta \mu \theta_{0}, V\left(x_{i, 1}\right)=\beta^{2} V_{o}, V\left(x_{i, 2}\right)=\beta^{2} \mu^{2} V_{o}$, $\operatorname{Cov}\left(x_{i, 1}, y_{i, 1}\right)=\beta \alpha_{1} V_{o}$ and $\operatorname{Cov}\left(x_{i, 2}, y_{i, 2}\right)=\beta \alpha_{2} V_{o}$. Note that the parameter $\mu$ enters both in the mean and the variances. The parameter $\mu$ is identified from the variances of demand over the two periods, taking the square root $\mu=\sigma_{x_{i, 2}} / \sigma_{x_{i, 1}}$. Now subtract the demand over the two periods $E\left(x_{i, 2}\right)-E\left(x_{i, 1}\right)=\alpha_{2}-\alpha_{1}-\beta \theta_{o}(\mu-1)$ in order to eliminate the individual effects and divide the covariance between demand and expenditure over the two periods $\operatorname{Cov}\left(x_{i, 1}, y_{i, 1}\right) / V\left(x_{i, 1}\right)=\alpha_{1} / \beta$ and $\operatorname{Cov}\left(x_{i, 2}, y_{i, 2}\right) / V\left(x_{i, 2}\right)=\alpha_{2} / \beta$. It follows that $\alpha_{2} / \alpha_{1}=\left(\operatorname{Cov}\left(x_{i, 2}, y_{i, 2}\right) V\left(x_{i, 1}\right)\right) /\left(\operatorname{Cov}\left(x_{i, 1}, y_{i, 1}\right) V\left(x_{i, 2}\right)\right)$. Now, from the mean of demand in first period, one gets $-\beta \theta_{o}=E\left(x_{i, 1}\right)-\alpha_{1}$ and expected demand variation is $E\left(x_{i, 2}\right)-E\left(x_{i, 1}\right)=\alpha_{2}-\alpha_{1}+\left(E\left(x_{i, 1}\right)-\alpha_{1}\right)(\mu-1)$. Using these two last conditions, we determine the values of $\alpha_{1}$ and $\alpha_{2}$ :

$$
\begin{aligned}
\alpha_{1} & =\frac{E\left(x_{i, 2}\right)-\left(\sigma_{x_{i, 2}} / \sigma_{x_{i, 1}}\right) E\left(x_{i, 1}\right)}{\frac{\operatorname{Cov}\left(x_{i, 2}, y_{i, 2}\right) / V\left(x_{i, 2}\right)}{\operatorname{Cov}\left(x_{i, 1}, y_{i, 1}\right) / V\left(x_{i, 1}\right)}-\frac{\sigma_{x_{i, 2}}}{\sigma_{x_{i, 1}}}} \\
\alpha_{2} & =\frac{E\left(x_{i, 2}\right)-\left(\sigma_{x_{i, 2}} / \sigma_{x_{i, 1}}\right) E\left(x_{i, 1}\right)}{1-\frac{\operatorname{Cov}\left(x_{i, 2}, y_{i, 2}\right) / V\left(x_{i, 2}\right)}{\operatorname{Cov}\left(x_{i, 1}, y_{i, 1}\right) / V\left(x_{i, 1}\right)} \frac{\sigma_{x_{i, 1}}}{\sigma_{x_{i, 2}}}}
\end{aligned}
$$

The expressions of $\alpha_{1}$ and $\alpha_{2}$ are similar to the ones of examples 3.1, apart from the growth correcting effect. From (3), the price elasticity can be directly deduced. 


\section{Concluding Remarks}

In this paper, we present an empirical strategy that allows to identify the demand parameters of a simple structural model. Several issues may be then worth considering. First, instead of a single homogeneous product, the structural model must introduce a differentiated and/or several products that induce new conditions for identification. Second, the model delivers a linear demand. One may ask if the previous results about identification still hold in the non-linear case. Third, we can investigate the potential of this approach from real data. Fourth, one can consider endogenous supply. Finally, the identification of the structural parameters allows to analyze the quantitative implications and welfare effects that follow a policy change.

\section{References}

Brown, J. and Rosen, H. (1982) "On the Estimation of Structural Hedonic Price Models", Econometrica, 50, pp 765-769.

Ekeland I., Heckman J. and Nesheim L. (2002) : Identifying Hedonic Models, American Economic Review, 92(2), 304-309.

Ekeland I., Heckman J. and Nesheim L. (2003) : Identification and Estimation of Hedonic Models, forthcoming in Journal of Political Economy.

Fève, F., Fève, P. and Florens, J-P. "Attribute Choice and Structural Econometrics of Price Elasticity of Demand", mimeo Greamq-Idei.

Kahn, S. and Lang K. (1988) "Efficient Estimation of Structural Hedonic Systems", International Economic Review, 29, pp. 157-166.

\section{Appendix}

Proof of Proposition 1: First note that the parameters $\theta_{o}$ and $\gamma$ are only identified from equations the mean of demand and expenditure. So we discard these two parameters from identification study. The identification of $\widetilde{\varphi}=\left\{\alpha, \beta, V_{o}\right\}$ is thus based on the remained equations. The condition for identification relies on the rank of $f(\widetilde{\varphi})$ where

$$
f(\widetilde{\varphi})=\left(\begin{array}{c}
\beta^{2} V_{o}-V\left(x_{i}\right) \\
\alpha^{2} V_{o}-V\left(y_{i}\right) \\
\beta \alpha V_{o}-\operatorname{Cov}\left(x_{i}, y_{i}\right)
\end{array}\right)
$$

Let the first derivative of $f(\widetilde{\varphi})$ with respect to $\widetilde{\varphi}$

$$
\frac{\partial f(\widetilde{\varphi})}{\partial \widetilde{\varphi}^{\prime}}=\left(\begin{array}{ccc}
0 & 2 \beta V_{0} & \beta^{2} \\
2 \alpha V_{o} & 0 & \alpha^{2} \\
\beta V_{o} & \alpha V_{o} & \alpha \beta
\end{array}\right)
$$

The determinant of $\partial f(\widetilde{\varphi}) / \partial \widetilde{\varphi}^{\prime}$ is zero and thus $f(\widetilde{\varphi})$ is not full rank. It follows that the condition for identification is not fulfilled. This completes the proof. 